\title{
A block-based background model for moving object detection
}

\author{
Omar Elharrouss, Abdelghafour Abbad, Driss Moujahid \\ Jamal Riffi and Hamid Tairi \\ LIIAN Laboratory, Department of Informatics Faculty of Sciences Dhar-Mahraz \\ University of Sidi Mohamed Ben Abdellah \\ P.B 1796 Atlas,Fez, Morocco
}

Received 22nd Jan 2016; accepted 1st Dec 2016

\begin{abstract}
Detecting the moving objects in a video sequence using a stationary camera is an important task for many computer vision applications. This paper proposes a background subtraction approach. As first step, the background is initialized using the block-based analysis before being updated in each incoming frame. Our background frame is generated by collecting the blocks background candidates. The block candidate selection is based on probability density function ( $p d f)$ computation. After that, we compute the absolute difference between the background frame and each frame of sequence. A noise filter is applied using the Structure/Texture decomposition in order to minimize the noise caused by background subtraction operation. The binary motion mask is formed using an adaptive threshold that was deduced from the weighted mean and variance calculation. To assure the correspondence between the current frame and the background frame, an adaptation of background model in each incoming frame is realized. After comparing results obtained from the proposed method to other existing ones, it was shown that our approach attains a higher degree of efficacy.
\end{abstract}

Key Words: Motion detection, Background subtraction, Background model, Background update, Video surveillance.

\section{Introduction}

Motion detection is a paramount study field of computer vision. Its purpose is to extract the moving objects at time $t$ in a video captured using a stationary camera. The motion detection is used for many applications. Among these applications there are video surveillance, human-machine interaction, the recognition of sign language specific to robotics applications and many others.

The approaches mainly used for motion detection can be classified into three categories: the time difference methods, the analysis of optical flow, and the background subtraction methods. For the first one, the time difference being the calculation of the difference between two or more consecutive images in order to extract the moving area [1], [2], but the problem in this approach is that the detected objects are incomplete and poorly presented. The second approach is the calculation of the optical flow [3],[4] which provides all information about the movement, but the real-time implementation is difficult and calculation of

Correspondence to: elharrouss.omar@gmail.com

Recommended for acceptance by Filiberto Pla

http://dx.doi.org/10.5565/rev/elcvia.855

ELCVIA ISSN: $1577-5097$

Published by Computer Vision Center / Universitat Autonoma de Barcelona, Barcelona, Spain 
flow being generally slow. The background subtraction approaches start by modeling the background(model of static scene), it can detect the most precise foreground[5-6] [24-31], , but it has many limitations like sensor noise (noise of acquisition and digitization) and management of homogeneous areas when the luminance difference between two moments is less than a threshold.

Most motion detection algorithms that exist in literature are presented as background subtraction methods, they generally have four steps. The main step is to develop a model of the background which contains the static part of the scene and then the absolute difference is calculated by comparing every frame of the sequence to the background frame. In order to extract the moving objects area called foreground a threshold operation is applied. To assert a best detection during all time of video sequence, the background must be updated in each incoming frame. All these methods require handling the following limitations which are the principle difficulties of motion detection based on background subtraction:

- Acquire a best background frame which contains just the static components of the scene.

- The environmental changes in the scene.

- The noise, in each incoming frame (noise of acquisition and digitization) or in the background subtraction results.

- The new object that can be installed a long time in the scene.

- Management of homogeneous areas when the luminance difference between two moments is less than a threshold.

Many approaches are proposed to overcome these constraints. For example, to handle the noise problem, Huang et al. [18] proposes a noise filter based on Bezier curve in order to smooth the noise pixels. To generate an accurate background model for motion detection and to reduce the influence of environmental changes in the scene, Cheng et al. [19] propose an illumination-sensitive background modeling approach based on an illumination evaluation. The selection of the best threshold represents a critical case in the segmentation step, when the luminance difference between two moments is less than this threshold. Many algorithms have been proposed.

In this paper, a background subtraction approach is proposed. Using the block-based and pixel-based processes, a background model is developed. Afterwards, the absolute difference between the background model and each incoming frame is calculated. Then a noise filter is applied on the background subtraction result in order to reduce the noise. The binary image of moving objects is generated using an adaptive threshold. In each incoming frame the background will be updated. The organization of the proposed approach is as following:

1. Each frame of the video sequence will be divided into blocks before collecting each one of them in a matrix of blocks. Then, for each matrix we compute the probability density function $(p d f)$ of each pixel. In order to determine the block background candidate, we compute the summation of $p d f s$ in each block. The block that has the maximum value of this summation is considered as block background candidate. After the selection of block background of each matrix, these blocks are combined to construct the background frame.

2. To reduce the noise in the results of the background subtraction the structure-texture decomposition [7] is applied on the absolute difference image. Just the structure component that contains just the homogeneous parts of the image is used in the segmentation step.

3. To detect the moving objects, a binary image is formed using a threshold deduced from the calculating the weighted mean and weighted variance of the absolute difference results.

4. In order to make the correspondence between the current frame and the background frame also to assure a best detection of moving objects, an adaptation of background model in each incoming frame is realized.

From the compared results of the proposed method and other methods in literature, our approach is effective in terms of quality and quantity of the detected moving objects. The rest of this paper will be conducted as follow: section 2 presents many related works of motion detection. In section 3 our approach will be presented in detail. Experimental results will be shown in section 4. Section 5 will contain our concluding remarks. 


\section{Related works}

Motion detection in a video sequence is an important task for motion analysis. Different methods are explored in this context. Most of them are classified as background subtraction methods [8-12],[14-29]. Where, the Background Modeling is the principal step in these different methods. Obtain a best background frame which contains just the static components of the scene, environmental changes in the scene, noise in each incoming frame in the video, new object that can be installed a long time in the scene, and the threshold selection are the principal constraints of motion detection methods based on background subtraction. In the literature, several approaches are proposed in the goal to obtain an accurate detection and to handle the difficulties cited above.

The basic methods of motion detection based on background subtraction use the time difference between the current frame and the previous one which considered as the background frame [8]. The detection results are represented by the pixels of the motion area that change the luminance between the two consecutive images. This approach is fast in term of computation time, but the difference is sensitive to noise, and the objects are badly detected particularly in the slow motion. To avoid these problems, some methods use the absolute difference between the current image and an image of the static scene (reference image). Thereafter, the binary-motion-detection-mask is computed using the segmentation process.

Several approaches have been proposed to generate a background model. The idea is to build a background model and to update it for each incoming frame. The algorithm that is based on temporal median filter with Exponentially Weighted Moving Average filtering effectively implements a temporal mode operation (TMF) [9]. In addition, this method statistically computes the temporal median value for each pixel in order to generate the adaptive background model. In the same context, the methods based on the statistical calculation have been developed [10],[11]. In [10], the background model is generated by computing the mean of the images in a time interval of video sequence. In [11], the weighted mean is used to develop the background model. For each pixel, a threshold is also represented by the standard deviation in the same interval to decide if the pixel belongs to the moving object or to the background.

In [12] a block alarm module efficiently determines background blocks within each frame. A unique twostage background training procedure is performed using Rough Training followed by Precise Training in order to generate a high-quality adaptive background model. In [13], first each frame is divided to blocks, and blocks in frame sequences are sorted to make block series. Finally PCA process is applied to these block series. Based on the PCA theorem, if there is change in block series which means there is not pure background, the main component of the block series is comparable to other components of the series. This approach was known as multi block PCA which was used before for detection changes in images.

In order to overcome the illumination change, background image must be updated in each image. The running average (RA) is commonly used to update the background model [14]. Then Different color spaces have been tested to improve the insensitivity of the detection to the illumination changes. In [15], the traditional RA method is modified to develop the adaptive background in the DCT domain. The fuzzy theory is used to generate the background model. For fuzzy background modeling, fuzzy running average is suggested to improve performance of running average method [16]. Running average method and its modified version are both simple and fast for background modeling. Because of low computational complexity and high memory compactness, the running average method is used in real-time systems.

The background estimation algorithm with $\Sigma-\Delta$ method is another method of background modeling (SDE) [17]. To estimate the background value the 'sgn' function is exploited. At every frame of the sequence, the estimation of background value is simply incremented by 1 if it's smaller than the input image value or decremented by 1 if it's greater than it. 


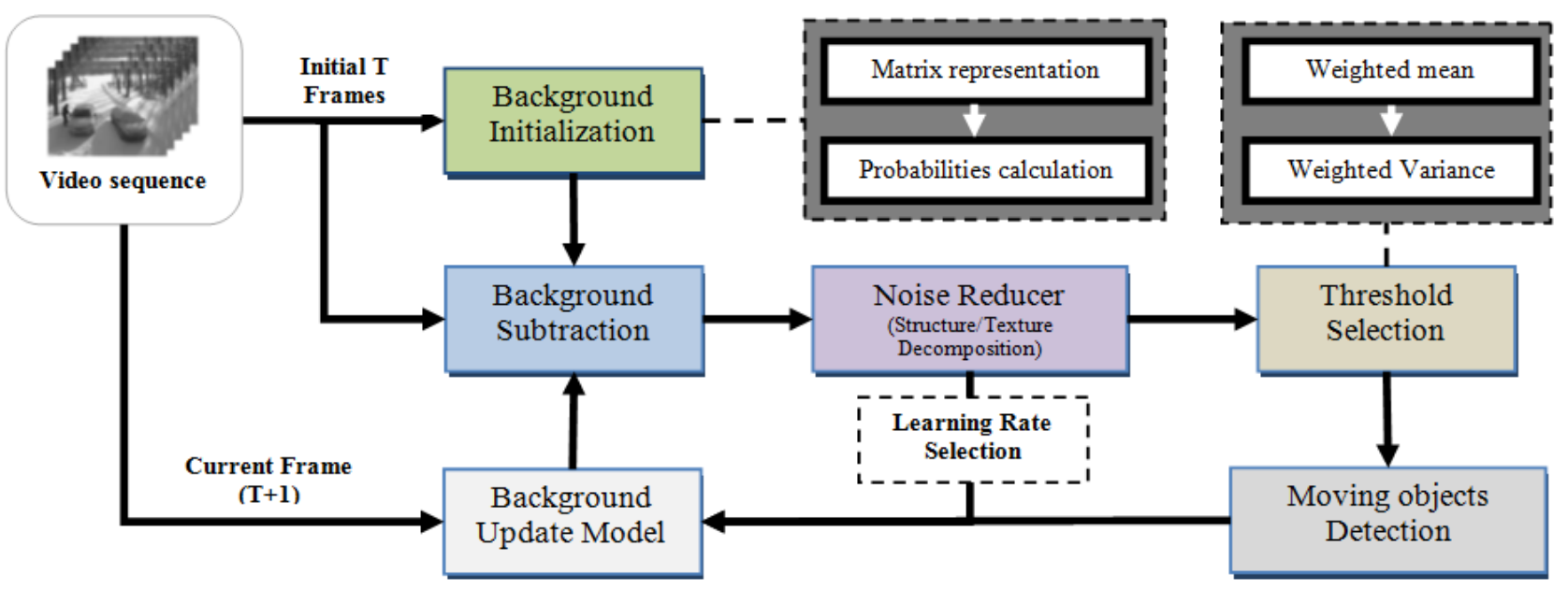

Fig. 1. Block diagram of the proposed background subtraction approach.

The 'sgn' function is also used to compute the time-variance representing the measure of the motion activity to decide if the pixel belongs to the background or to the moving object. Finally, the detection label is developed by the comparison between the time-variance value and the absolute difference value.

Another method provides a pyramidal structure for motion detection (MDPS) [18]. The proposed method uses the spatial and temporal characteristics to generate a pyramidal structure of the background model. This background model has been modified by the best background candidates based on the background blockmarching framework. In order to refine the background subtraction results, the noise reducer method is exploited using the Bezier curve smoothing method. As a finale step, a threshold function based on Probability Mass Function (PMF) and Commutative Distribution Function (CDF) was proposed to compute the binary image of moving objects.

In the same context an illumination-sensitive background modeling approach is proposed in order to analyze the illumination change in each incoming frame and then detect moving objects (ISBS) [19]. The background generation uses two background candidates, including a light background image and a dark background image in order to update the background model. Based on illumination evaluation and the background model, a thresholding function is proposed to generate the binary mask of moving object.the approch handel the illumination change is the scene but the segmentation part is not effective.

In the goal to overcame the diffuculties of backgroundsubtraction methods, this paper proposes a background subtraction approach with two main advantages. First, it gives a best background initialization before updating it during each incoming frame of the sequence using an adaptive learning rate. Second, the noise reduction helps the determination of true foreground.

\section{Proposed method}

In this section, the proposed approach for moving objects detection in a video sequence is presented. The detection uses a video sequence, where the images are captured by a fixed camera installed in scenes which contain moving objects. Our approach is obtained in four steps: background initialization, background subtraction and noise reduction, segmentation of moving object and finally background update. Fig. 1 illustrates all proposed process for detecting moving objects in a video sequence. 


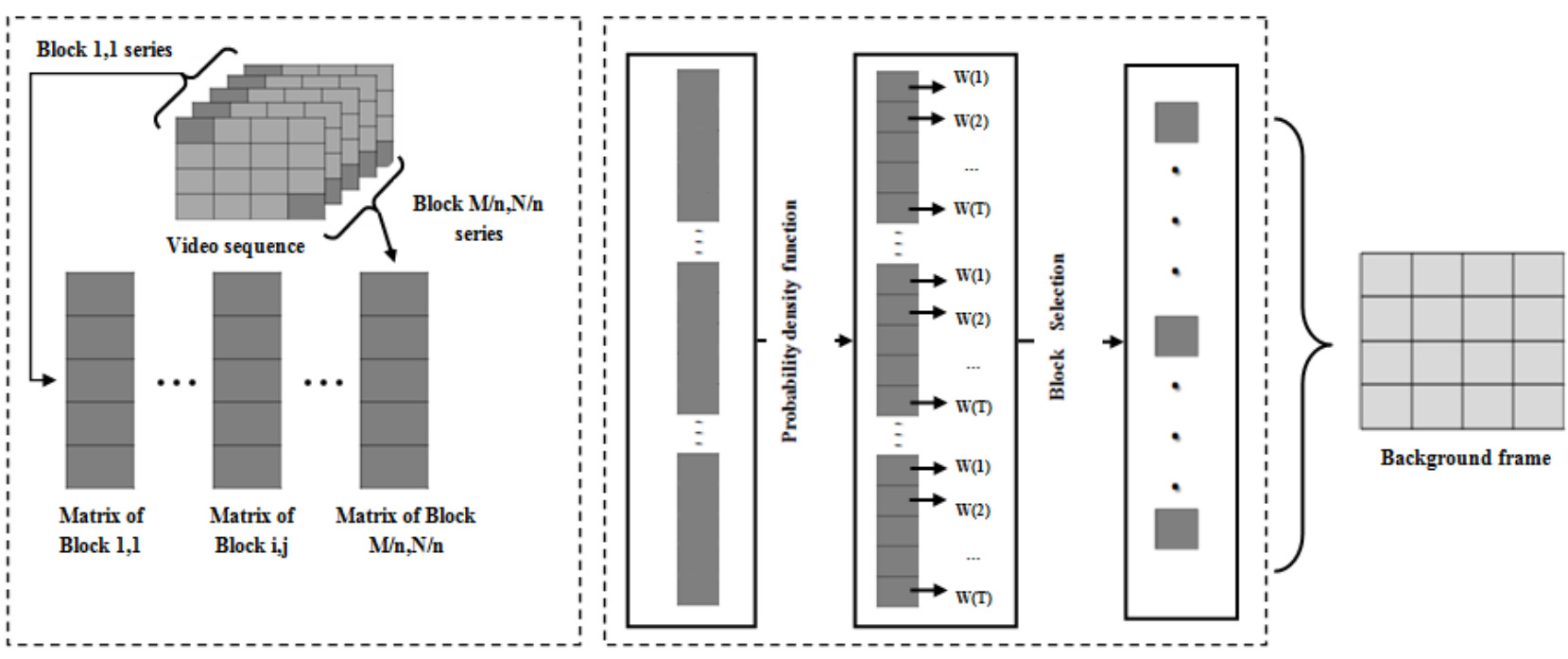

Fig. 2. Background model construction process.

\subsection{Background initialization}

The background model is an image that the pixels retain its value for a long period or throughout all time of the video. Based on this theory, the proposed background initialization involves two important modules including matrix representation module and block background selection module.

1) Matrix representation: Our background model initialization consists in dividing the $T$ initial images of the sequence into blocks of $n \times n$ pixels where $\mathrm{n}$ can be in the range of $\{2,4,8,16, .$.$\} . In this paper, we$ use $n=16$. Therefore, each formed block has $n \times n$ pixels. After that, each block $(i, j)$ of the sequence are combined to form a matrix $M b_{i, \mathrm{j}} . M b_{i, \mathrm{j}}$ Is the matrix of all pixels of the blocks $b_{i, \mathrm{j}}$ of the sequence. Consequently, we have $M \times N / \mathrm{n}^{2}$ matrix of blocks, such as each image of the sequence has $M$ rows and $N$ columns. The matrix can be expressed as follows:

$$
M b_{i, j}=\left[\begin{array}{c}
b_{i, j, 1}(x, y) \\
\vdots \\
b_{i, j, t}(x, y) \\
\vdots \\
b_{i, j, T}(x, y)
\end{array}\right], t=1,2, \ldots, T
$$

Where T represents the number of image sequence, $i \in\{1,2, \cdots, \mathrm{M} / \mathrm{n}\}$ and $j \in\{1,2, \cdots, \mathrm{N} / \mathrm{n}\}$.

2) Block background selection: After the matrixes are formed, the probability density function ( $p d f)$ of pixels intensity of each matrix is calculated. The probability density function of each pixel luminance $l$ of the matrix $M b_{i, j}$ can be expressed as follows:

$$
p d f(l)=n_{l} /(T \times n)
$$

The pixel that has the highest value of $p d f$ is the pixel in the sequence shown. The same idea is applied to find the block background candidate. The background block is the block that's more pixels displayed in sequence. In order to select the block background, the probability of each block in the matrix represented by the sum of $p d f$ s of each pixel is computed in equation (3). The probability of block at time $t$ can be expressed as follows:

$$
W_{i, j}(t)=\sum_{l \in b_{i, j}(t)} p d f(l)
$$


The block that has the highest value of $W_{i, j}$ is classified as a block background. Fig. 2 illustrates the process of construction of the background frame. After the selection of the blocks background candidate that has the highest value of probability in the matrix. A background frame is formed by collecting all blocks candidates in their own positions. This model is used for $\mathrm{T}$ initial frames of the sequences and would be updated after each incoming frame. By the proposed technique we can generate a background frame with the real pixels value which still invariant during most of the time of the video sequence. Algorithm 1 represents the proposed process. From the experimental results, our approach can generate a background image without the ghost effect that can produces false detection. Also the noise or partial change in pixels ignored while other methods is somehow sensitive to partial noise in pixels intensity.

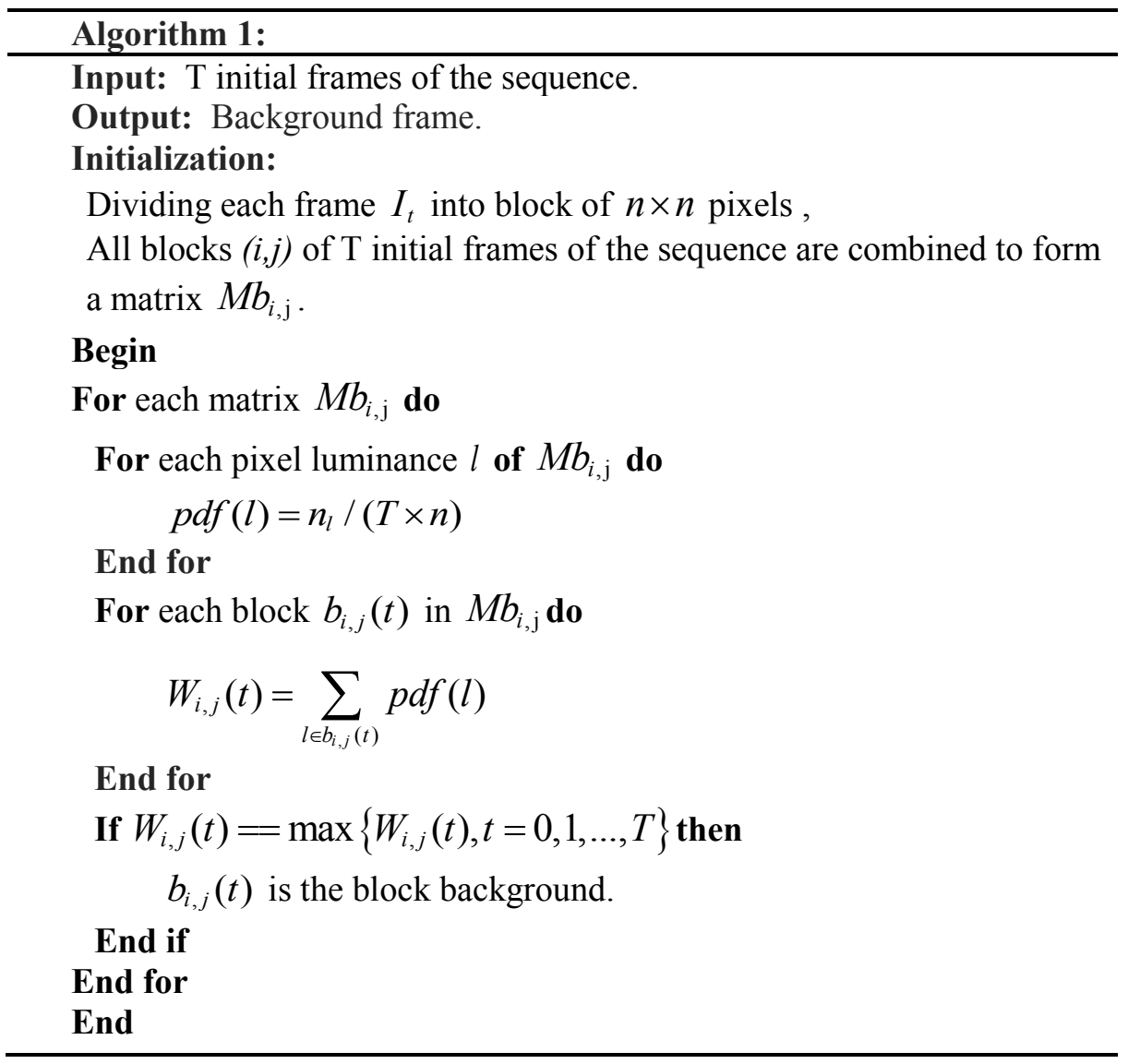

\subsection{Background subtraction and noise minimization}

After the background frame generation the background subtraction is used by computing the absolute difference $\Delta_{t}$ between each frame $I_{t}$ of the video sequence and the developed background frame:

$$
\Delta_{t}(x, y)=\left|B_{t}(x, y)-I_{t}(x, y)\right|
$$

During the background subtraction processes, each image of the sequence can be full of noise. In addition, a pixel of the background frame can be falsely modeled. Thus, in the absolute difference image this pixel will be presented as noise. Our purpose is to remove this noise before starting the thresholding phase. The structure-texture decomposition method proposed by the Osher and Vese [7] is used. It a technique to split a given image $f$ into $S+T(f=S+T)$ of a bounded variation component (Structure) and a component that contains the oscillating part (Texture/Noise) of the image. We applied this technique on the absolute difference image. Then, we use just the structure component which contains just the homogeneous parts of 
the image. Automatically we ignore the second component that contains the texture and noise. The structure component of $\Delta_{t}$ is used to generate the moving the moving object detection mask.

$$
\Delta_{t}^{\prime}=\text { Structure_component }\left(\Delta_{t}\right)
$$

The Fig. 3 Illustrate the background subtraction and the noise minimization phase.

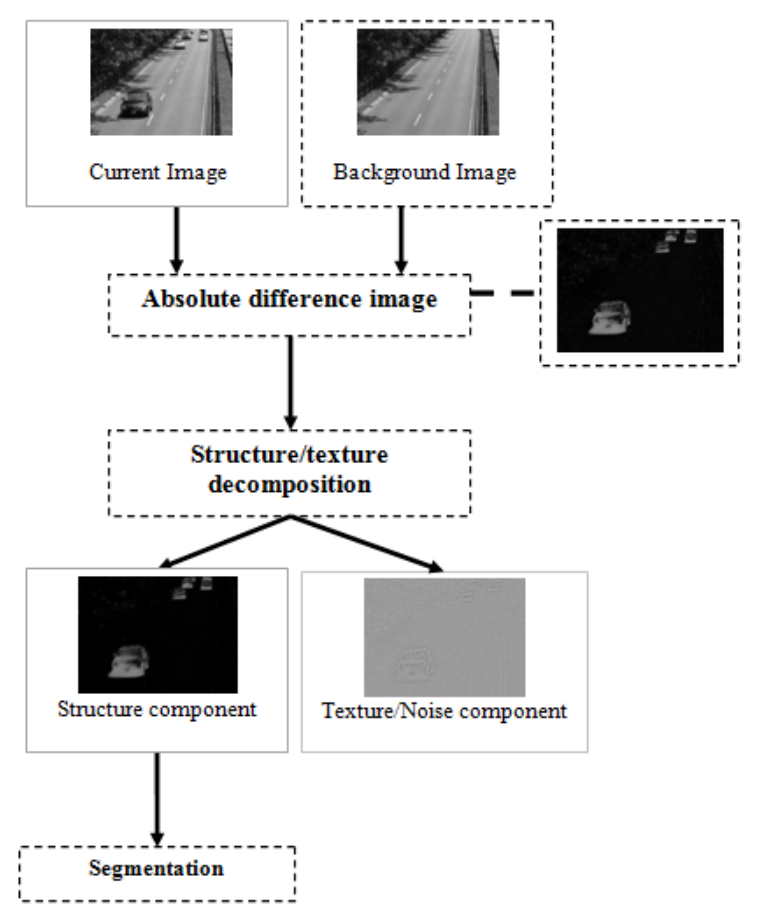

Fig. 3. Background subtraction and noise filter phase using Structure/Texture decomposition.

\subsection{Moving objects detection}

When the background image is generated, the absolute difference between the current image in the sequence and the background image is calculated. We can observe the regions of the gray-level in this obtained result. To distinguish the background region from the foreground region, a threshold operation is applied, but the selection of the best threshold can be difficult. Most of the algorithms select it by testing a set of threshold values and then choose the one that gives the best results. In this paper, we use the statistical characteristics of image obtained after the computation of the absolute difference and the noise reducer filter. The threshold is formed by calculating the weighted mean and variance. The weighted mean and the variance are described in the following equations:

$$
\begin{gathered}
\mu=\frac{\sum_{x} \sum_{y} W(x, y) \cdot \Delta_{t}^{\prime}(x, y)}{\sum_{x} \sum_{y} W(x, y)} \\
\sigma^{2}=\frac{\sum_{x} \sum_{y} W(x, y) \cdot\left(\Delta_{t}^{\prime}(x, y)-\mu\right)^{2}}{\sum_{x} \sum_{y} W(x, y)}
\end{gathered}
$$

Where $\Delta_{t}^{\prime}(x, y)$ presents the intensity of pixel $(x, y)$ of the structure component obtained from absolute difference in equation (5). In order to minimize the effect of outliers the weight $W(x, y)$ is used. 


$$
W(x, y)=\exp \left(\frac{\left(\Delta_{t}^{\prime}(x, y)-\operatorname{mean}\left(\Delta_{t}^{\prime}(\mathrm{p}, \mathrm{q})\right)\right)^{2}}{-2 S D}\right)
$$

Where $(p, q)$ is the location set of 8-connected neighboring pixels around each pixel $(x, y)$ of the image. The value of SD is taken to be 5 after the experimental evaluation.

The binary image of the moving objects in each image of the sequence is generated using the $\lambda \sigma$ defined in the interval of $[1,2]$. The moving objects detection mask $\mathrm{D}_{t}(x, y)$ is expressed as follows:

$$
D_{t}(x, y)=\left\{\begin{array}{cc}
1 & \text { if } \Delta_{t}^{\prime}(x, y)>\lambda \sigma \\
0 & \text { otherwise }
\end{array}\right.
$$

\subsection{Background update}

In order to make the correspondence between the current frame and the background frame, also to assure a best detection of moving objects, an adaptation of the background model in each incoming frame must be realized. Therefore, after the computation of the motion mask, a background frame is developed based on the current frame and the previous background frame. From the binary image of detected object any pixel that is classified as background will be updated in the new background frame by the combining of the previous background value and the current frame values. The idea is to adapt very quickly a pixel classified as background and very slowly a pixel classified as foreground. The expression of the background adaptation can be expressed as follows:

$$
B_{t}= \begin{cases}\alpha \cdot B_{t-1}+(1-\alpha) \cdot I_{t} & \text { if }(x, y) \text { is static } \\ (1-\alpha) \cdot B_{t-1}+\alpha \cdot I_{t} & \text { if }(x, y) \text { is moving }\end{cases}
$$

Where $\alpha$ is the learning rate parameter. The majority method generally selects this value before being used .So it is static throughout the sequences and for each pixel in each frame. In this paper, we purpose a dynamic value of $\alpha$ used in each pixel and formulated as follows:

$$
\alpha(x, y)=1-\exp \left(-\Delta_{t}^{\prime}(x, y)\right)
$$

The proposed background subtraction approach has been proposed in order to solve the most critical stage for moving objects detection approaches. A set of processes are followed to attain this objective, including background frame development process, noise reducer filter process, foreground detection process and finally the background updating process.
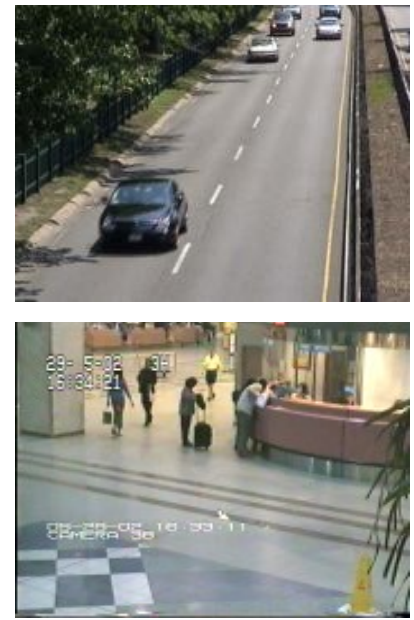
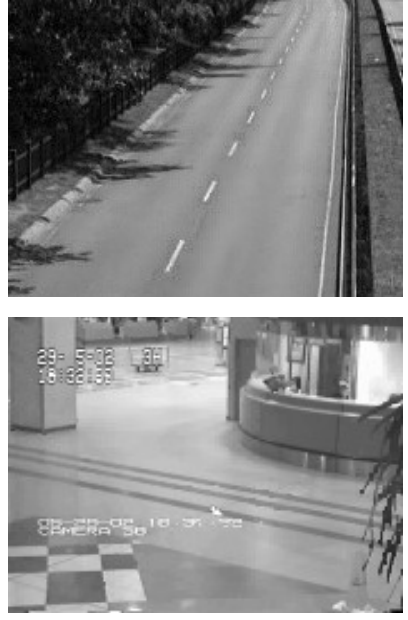
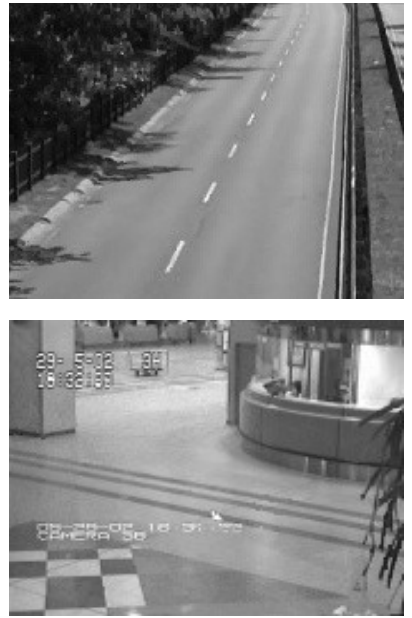

Fig. 4. Background images of "Highway" and "Airport" sequences, first column: an image of the sequence, second column: initial background model, third column: background model after 1000 frame. 


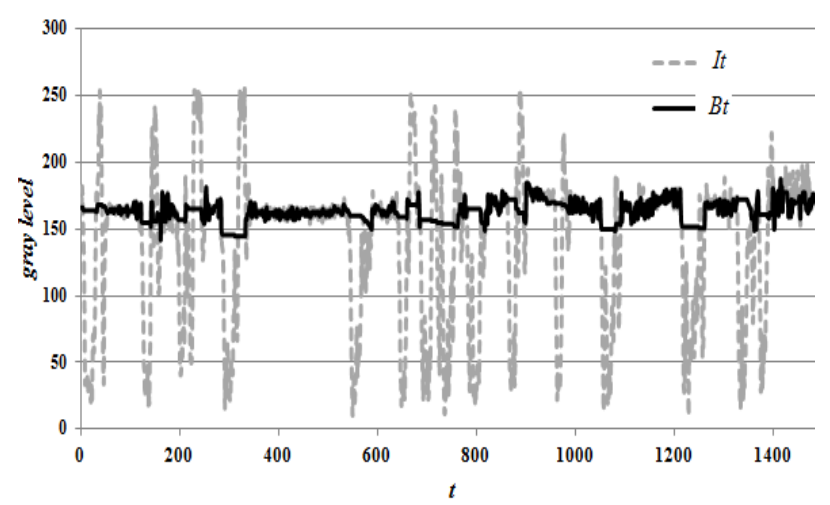

(a)

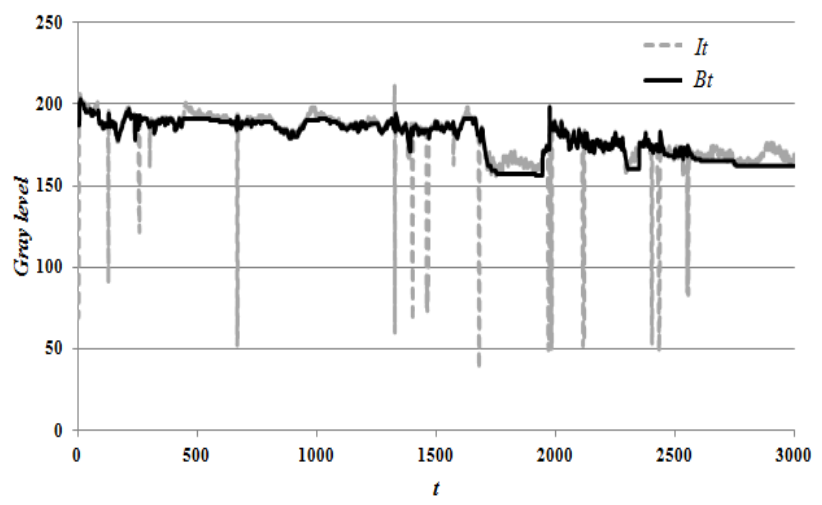

(b)

Fig. 5. A pixel history during all time of the sequence (a) Highway sequence ,(b) Airport sequence.

\section{Experimental results}

This section is designed to demonstrate the experimental results of our proposed approach. All tests are performed on two data sets ( $\operatorname{Star}^{1}$, and ChangeDetection ${ }^{2}$ ) captured by a camera installed in the public scenes like train stations or airports, or in the private scenes like offices. Those sequences contain different types of objects such as vehicles, human bodies and others, moving or waiting in the scene. We compare our results with four other background subtraction methods: (1) SDE [17]; (2) MDPS [18]; (3) ISBS [19]; and (4) CBMFE [27]. We implement the four methods, using the default values of all the parameters given in each paper. These methods are tested on the same video sequences described below.

The scene static modeling is the main step in motion detection methods based on background subtraction. Our proposed approach (PRO) uses the block-based analysis for initializing the background frame before update it for each incoming frame. The background model images of the "Highway" and "Airport" sequences generated by our proposed approach are presented in Fig. 4 that shows the background initialization image and background update image after 1000 frame. The background initial images are illustrated in Fig. 4(second column). We can easily remark that our approach can generate a background model without the 'ghost effect' and the erroneous generation of noise. In addition, it can give a best background initialization before it has been updated during the rest frames of the sequence as shown in Fig. 4 (third column).

The noise in each image of the sequence, thus the noise resulted from a false modeling of a pixel, are the critical stages for the moving objects segmentation. Also, the noise affects image quality that leads to the low quality of detection. To solve this problem, the structure/texture decomposition is used. To compute the objects detection mask, just the structure component is considered, automatically we ignoring the second component that contains the texture and noise. Fig. 6 represents the background subtraction frame before and after the noise reduction. Comparing the absolute difference image in Fig. 6(a), and his structure component image in Fig. 6(c) we can observe that the noise is reduced using the structure/texture decomposition. That also confirmed by the results in Fig. 6(b) and Fig. 6(d) which are the inverse values of the absolute difference image and the structure component image.

Fig. 7 illustrates the image sequences of ChangeDetection and Star data sets, ground truths, and binary mask of moving objects obtained by our proposed approach and the state-of-the-art methods. We choose three image sequences ChangeDetection data sets and eight image sequences from Star data set which contain different objects and most categories of video surveillance. Visually, we notice that our approach leads to a robust and efficient detection. This is confirmed by the quantitative results presented in Table I representing the similarity and the F-measure metrics of our approach along with the results in the figure 8 which shows the F-measure curves achieved for each sequence.

\footnotetext{
${ }^{1} \mathrm{http}: / /$ perception.i2r.a-star.edu.sg/bk_model/bk_index.html

${ }^{2}$ http://www.changedetection.net.
} 


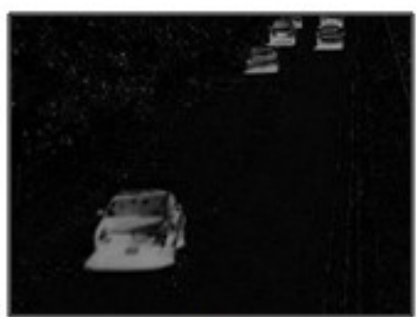

(a)

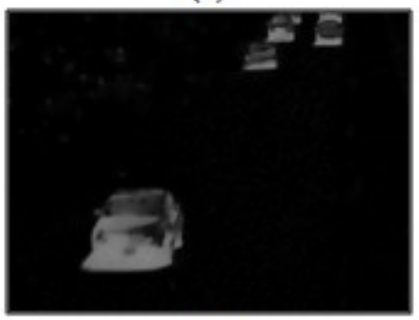

(c)

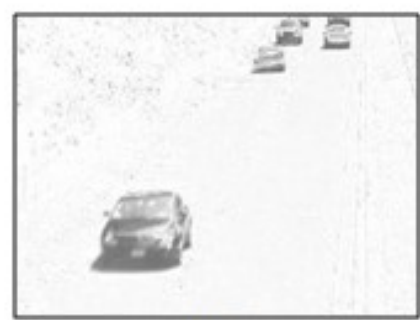

(b)

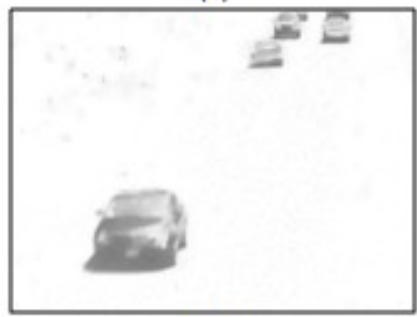

(d)

Fig. 6. Noise minimization filtre (a) absolute difference image, (b) inverse of (a), (c) the structure component image, (d) inverse of (c).

The motion detection results obtained by our proposed background subtraction approach are compared with those obtained through other state-of-the-art methods. For measuring the accuracy of the detection results in term of quality and quantity of the detected moving objects, different measures are used, such as Recall, Precision, F-measure, and Similarity. Recall gives the percentage of necessary positives via the compared total number of true positive pixels in the ground truth. Precision gives the percentage of unnecessary positives through the compared total number of positive pixels in the detected binary objects mask. F-measure, the weighted harmonic mean of Precision and Recall computes the quality of detection. Quantitative evaluation of detected objects is determined by the Similarity measure. These measures can be expressed as follows:

$$
\text { Recall }=\frac{t p}{t p+f n}
$$

where $t p$ is the total number of true positive pixels in the motion mask, $f n$ is the total number of false negative pixels in the motion mask, and $(t p+f n)$ indicates the total number of true positive pixels in the ground truth.

$$
\text { Precision }=\frac{t p}{t p+f p}
$$

Where $f p$ is the total number of false positive pixels in the motion mask and $(t p+f p)$ indicates the total number of positive pixels in the detected motion mask.

$$
\begin{gathered}
F-\text { measure }=\frac{2 * \text { Recall } * \text { Precision }}{\text { Recall }+ \text { Precision }} \\
\text { Similarity }=\frac{t p}{t p+f p+f n}
\end{gathered}
$$

The proposed approach thus the state-of-the-art methods are applied on six video sequences in representation of typical situations which are critical for video surveillance systems. Fig. 7 illustrates the experimental results of moving object detection. It represent binary image of the moving objects thus the original frames and the ground truth frames. These results were generated by SDE [17], MDPS [18]; ISBS [19], (4) CBMFE [27] and PRO methods. For almost every video sequence, we can observe that the generated binary objects mask of the our approach is more precise than those obtained the other methods. 


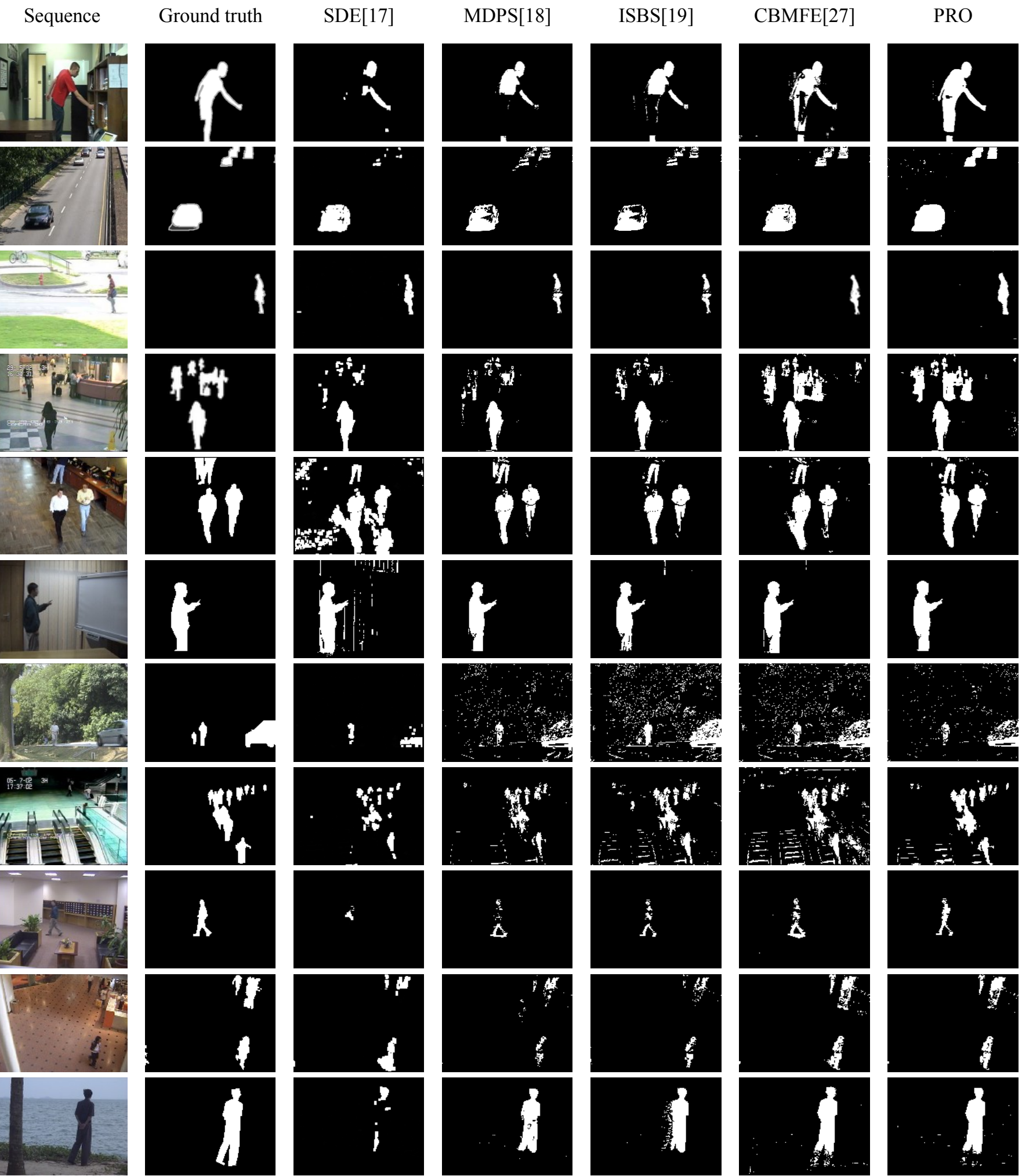

Fig. 7. Objects detection, first column: an image of the sequence, second column: the groundtruth, third column:SD[17] results, fourth column:MDPS [18] results, fifth column: ISBS[19] results, sixth column: CBMFE[27] results, lastcolumn: proposed approach results. 


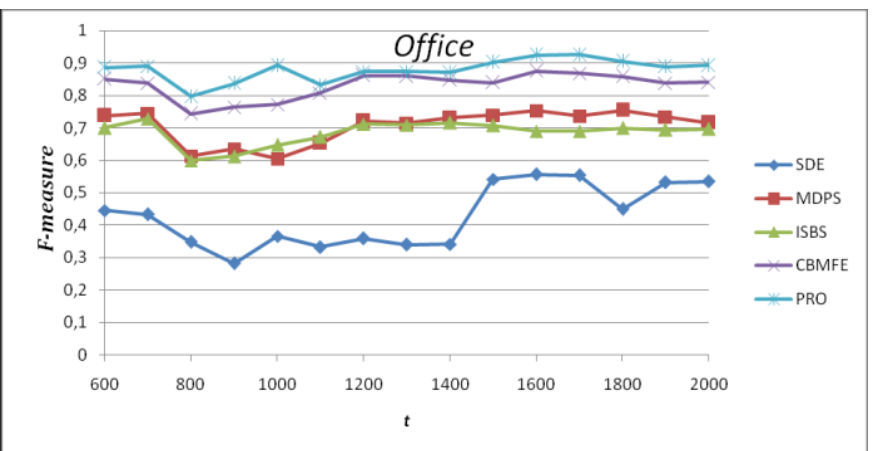

(a)

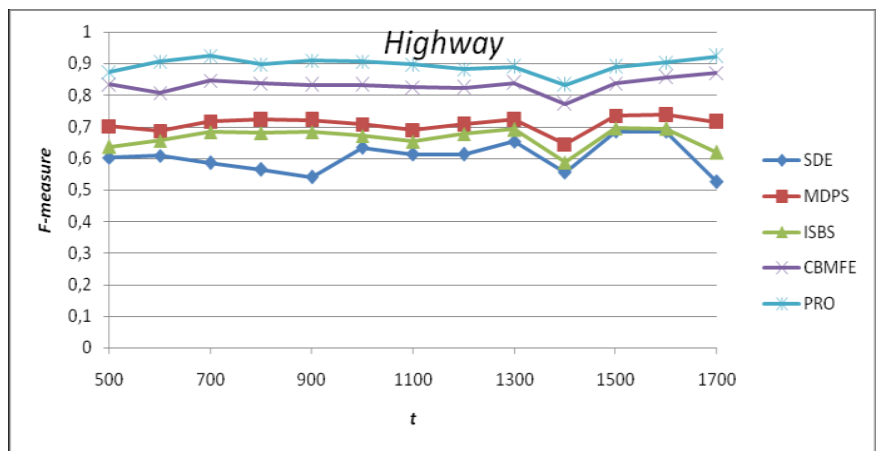

(b)

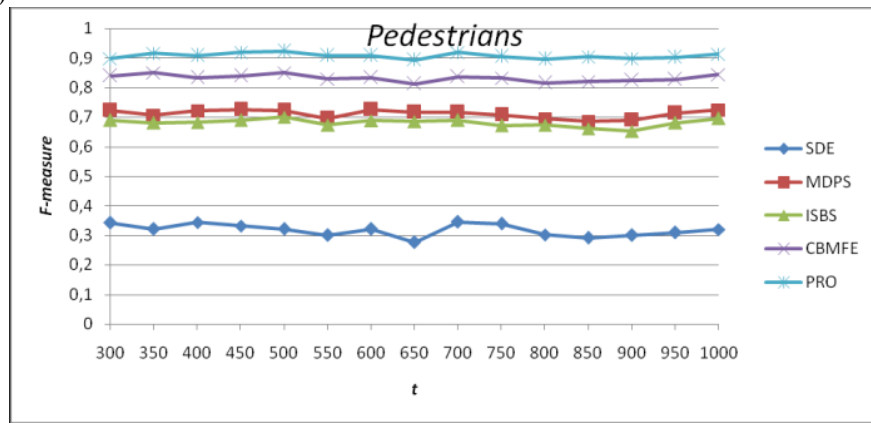

(c)

Fig. 8. F-measure curves achieved for: (a) Office sequence, (b) Highway sequence, (c) Pedestrians sequence.

\begin{tabular}{|l|c|c|c|c|c|c|}
\hline Sequence & Evaluation & SDE[17] & MDPS[18] & ISBS[19] & CBMFE[27] & PRO \\
\hline Office & Similarity & 0.224 & 0.431 & 0.472 & 0.632 & $\mathbf{0 . 8 1 1}$ \\
& F-measure & 0.366 & 0.602 & 0.641 & 0.774 & $\mathbf{0 . 8 9 6}$ \\
\hline Highway & Similarity & 0.316 & 0.596 & 0.544 & 0.759 & $\mathbf{0 . 8 4 2}$ \\
& F-measure & 0.481 & 0.746 & 0.705 & 0.862 & $\mathbf{0 . 9 1 4}$ \\
\hline Pedestrians & Similarity & 0.160 & 0.562 & 0.520 & 0.688 & $\mathbf{0 . 8 0 5}$ \\
& F-measure & 0.276 & 0.719 & 0.684 & 0.815 & $\mathbf{0 . 8 9 2}$ \\
\hline Airport & Similarity & 0.469 & 0.490 & 0.538 & 0.674 & $\mathbf{0 . 7 2 3}$ \\
& F-measure & 0.638 & 0.657 & 0.700 & 0.805 & $\mathbf{0 . 8 3 9}$ \\
\hline Bootstrap & Similarity & 0.213 & $\mathbf{0 . 7 3 6}$ & 0.693 & 0.654 & 0.687 \\
& F-measure & 0.351 & $\mathbf{0 . 8 4 8}$ & 0.818 & 0.791 & 0.814 \\
\hline Curtain & Similarity & 0.462 & 0.871 & 0.768 & 0.860 & $\mathbf{0 . 9 1 9}$ \\
& F-measure & 0.632 & 0.931 & 0.869 & 0.925 & $\mathbf{0 . 9 5 8}$ \\
\hline Campus & Similarity & 0.172 & 0.296 & 0.384 & 0.354 & $\mathbf{0 . 4 2 7}$ \\
& F-measure & 0.294 & 0.457 & 0.555 & 0.523 & $\mathbf{0 . 5 9 9}$ \\
\hline Escalator & Similarity & 0.230 & 0.413 & 0.406 & 0.426 & $\mathbf{0 . 5 8 1}$ \\
& F-measure & 0.375 & 0.585 & 0.577 & 0.597 & $\mathbf{0 . 7 3 5}$ \\
\hline Lobby & Similarity & 0.212 & 0.354 & 0.526 & 0.449 & $\mathbf{0 . 5 4 5}$ \\
& F-measure & 0.350 & 0.523 & 0.690 & 0.620 & $\mathbf{0 . 7 0 6}$ \\
\hline Shopping & Similarity & 0.329 & 0.273 & 0.301 & 0.589 & $\mathbf{0 . 6 2 3}$ \\
Mal & F-measure & 0.495 & 0.429 & 0.462 & 0.741 & $\mathbf{0 . 7 6 7}$ \\
\hline Water & Similarity & 0.125 & 0.718 & 0.652 & $\mathbf{0 . 8 0 4}$ & 0.785 \\
Surface & F-measure & 0.223 & 0.836 & 0.789 & $\mathbf{0 . 8 9 1}$ & 0.879 \\
\hline
\end{tabular}

Table 1: The F-measure and the Similarity values for each method.

In order to assert the results represented by the binary image in Fig. 7, the qualitative and quantitative results for one test frame of each video sequence obtained by each method are represented in Table 1 . We can observe that, for almost every test sequence, the generated binary image of our proposed approach is more precise than those obtained by the SDE [17], MDPS [18], ISBS [19] and CBMFE [27] methods. The proposed method attains the highest quantitative and qualitative values such as the accuracy rate is over $72 \%$ for Similarity measures and over than $82 \%$ F-measure values in the almost sequences. 


\section{Conclusion}

We have presented a background subtraction approach which tested to handle a variety of critical situations for moving objects detection. As first step, a background model is formed based on a block-based process. Then a noise minimizing filter is applied on the subtraction results using the Structure/Texture Decomposition. The weighted mean and variance are used to segmenting the moving objects in each frame of the sequence. To assert a best detection during all time of the video, a background updating procedure is proposed. There are two main advantages of proposed approach. First, it gives a best background initialization before updating it during each incoming frame of the sequence using an adaptive learning rate. Second, the noise reduction helps the determination of true foreground. From the visualized results thus the quantitative and qualitative measurements, our proposed approach is effective in terms of quality and quantity of the detected moving objects.

\section{References}

[1] G. L. Foresti, V. Murino and C. Regazzoni, "Vehicle recognition and tracking from road image sequences," in IEEE Transactions on Vehicular Technology, vol. 48, no. 1, pp. 301-318, Jan 1999. DOI: $10.1109 / 25.740109$.

[2] W. Shuigen, C. Zhen and D. Hua, "Motion Detection Based on Temporal Difference Method and Optical Flow field," 2009 Second International Symposium on Electronic Commerce and Security, Nanchang, 2009, pp. 85-88. doi: 10.1109/ISECS.2009.62.

[3] LI, Jin-zong, YUAN, Lei, et LI, Dong-dong. Tracking method of moving target based on detection of feature optical flow. Systems Engineering and Electronics, 2004, vol. 27, no 3, p. 422-426.

[4] Mahraz, M. A., Riffi, J., \& Tairi, H. (2014). Motion estimation using the fast and adaptive bidimensional empirical mode decomposition. Journal of Real-Time Image Processing, 9(3), 491-501. DOI: $10.1007 / \mathrm{s} 11554-012-0259-4$.

[5] HARITAOGLU, Ismail, HARWOOD, David, et DAVIS, Larry S. W 4: Real-time surveillance of people and their activities. Pattern Analysis and Machine Intelligence, IEEE Transactions on, 2000, vol. 22, no 8, p. 809-830. DOI: 10.1109/34.868683.

[6] LIU, Wei, YU, Hongfei, YUAN, Huai, et al. Effective background modelling and subtraction approach for moving object detection. IET Computer Vision, 2014, vol. 9, no 1, p. 13-24.

[7] VESE, Luminita A. et OSHER, Stanley J. Modeling textures with total variation minimization and oscillating patterns in image processing. Journal of scientific computing, 2003, vol. 19, no 1-3, p. 553572. DOI: $10.1023 / \mathrm{A}: 1025384832106$.

[8] LEUNG, Maylor K. et YANG, Yee-Hong. Human body motion segmentation in a complex scene. Pattern recognition, 1987, vol. 20, no 1, p. 55-64. DOI:10.1016/0031-3203(87)90017-3.

[9] SHOUSHTARIAN, B. A practical approach to real-time dynamic background generation based on a temporal median filter. Journal of Sciences, Islamic Republic of Iran, 2003, vol. 14, no 4, p. 351-362.

[10] ORAL, Mustafa et DENIZ, Umut. Centre of mass model-A novel approach to background modelling for segmentation of moving objects. Image and Vision Computing, 2007, vol. 25, no 8, p. 13651376.DOI: 10.1016/j.imavis.2006.10.001.

[11] AKULA, Aparna, KHANNA, Nidhi, GHOSH, Ripul, et al. Adaptive contour-based statistical background subtraction method for moving target detection in infrared video sequences. Infrared Physics \& Technology, 2014, vol. 63, p. 103-109.DOI: 10.1016/j.infrared.2013.12.012.

[12] CHENG, Fan-Chieh, HUANG, Shih-Chia, et RUAN, Shanq-Jang. Advanced background subtraction approach using Laplacian distribution model. In :Multimedia and Expo (ICME), 2010 IEEE International Conference on. IEEE, 2010. p. 754-759. DOI: 10.1109/ICME.2010.5582598.

[13] GHAEMINIA, Mohammad Hossein et SHOKOUHI, Shahryar Baradaran. Adaptive background model for moving objects based on PCA. In : Machine Vision and Image Processing (MVIP), 2010 6th Iranian. IEEE, 2010. p. 1-4. DOI: 10.1109/IranianMVIP.2010.5941174. 
[14] EL BAF, Fida, BOUWMANS, Thierry, et VACHON, Bertrand. Fuzzy integral for moving object detection. In : Fuzzy Systems, 2008. FUZZ-IEEE 2008.(IEEE World Congress on Computational Intelligence). IEEE International Conference on. IEEE, 2008. p. 1729-1736. DOI: 10.1109/FUZZY.2008.4630604.

[15] WANG, Weiqiang, YANG, Jie, et GAO, Wen. Modeling background and segmenting moving objects from compressed video. Circuits and Systems for Video Technology, IEEE Transactions on, 2008, vol. 18, no 5, p. 670-681. DOI: 10.1109/TCSVT.2008.918800.

[16] SIGARI, Mohamad Hoseyn, MOZAYANI, Naser, et POURREZA, H. Fuzzy running average and fuzzy background subtraction: concepts and application.International Journal of Computer Science and Network Security, 2008, vol. 8, no 2, p. 138-143.

[17] MANZANERA, Antoine et RICHEFEU, Julien C. A new motion detection algorithm based on $\Sigma-\Delta$ background estimation. Pattern Recognition Letters, 2007, vol. 28, no 3, p. 320-328.DOI: 10.1016/j.patrec.2006.04.007.

[18] HUANG, Shih-Chia et CHENG, Fan-Chieh. Motion detection with pyramid structure of background model for intelligent surveillance systems. Engineering Applications of Artificial Intelligence, 2012, vol. 25, no 7, p. 1338-1348.DOI: 10.1016/j.engappai.2012.02.002.

[19] CHENG, Fan-Chieh, HUANG, Shih-Chia, et RUAN, Shanq-Jang. Illumination-sensitive background modeling approach for accurate moving object detection.Broadcasting, IEEE Transactions on, 2011, vol. 57, no 4, p. 794-801. DOI: 10.1109/TBC.2011.2160106.

[20] YANG, Bo, GUO, Yunlong, MING, Yangyang, et al. An Effective Background Subtraction under a Continuosly and Rapidly Varying Illumination. In : Future Networks, 2010. ICFN'10. Second International Conference on. IEEE, 2010. p. 16-19. DOI: 10.1109/ICFN.2010.17.

[21] ELGAMMAL, Ahmed, DURAISWAMI, Ramani, HARWOOD, David, et al.Background and foreground modeling using nonparametric kernel density estimation for visual surveillance. Proceedings of the IEEE, 2002, vol. 90, no 7, p. 1151-1163. DOI: 10.1109/JPROC.2002.801448.

[22] GAO, Zhi, CHEONG, Loong-Fah, et WANG, Yu-Xiang. Block-sparse RPCA for salient motion detection. Pattern Analysis and Machine Intelligence, IEEE Transactions on, 2014, vol. 36, no 10, p. 1975-1987. DOI: 10.1109/TPAMI.2014.2314663.

[23] REDDY, Veerababu, SANDERSON, Conrad, et LOVELL, Brian C. Improved foreground detection via block-based classifier cascade with probabilistic decision integration. Circuits and Systems for Video Technology, IEEE Transactions on, 2013, vol. 23, no 1, p. 83-93. DOI: 10.1109/TCSVT.2012.2203199.

[24] YEH, Chia-Hung, LIN, Chih-Yang, MUCHTAR, Kahlil, et al. Real-time background modeling based on a multi-level texture description. Information Sciences, 2014, vol. 269, p. 106-127.DOI: 10.1016/j.ins.2013.08.014.

[25] LAUMER, Marcus, AMON, Peter, HUTTER, Andreas, et al. Compressed domain moving object detection by spatio-temporal analysis of H. 264/AVC syntax elements. In : Picture Coding Symposium (PCS), 2015. IEEE, 2015. p. 282-286. DOI: 10.1109/PCS.2015.7170091.

[26] Hong Han; Jianfei Zhu; Shengcai Liao; Zhen Lei; Li, S.Z., "Moving Object Detection Revisited: Speed and Robustness," Circuits and Systems for Video Technology, IEEE Transactions on, vol.25, no.6, p.910,921. DOI: 10.1109/TCSVT.2014.2367371.

[27] Yong Wang, Qian Lu, Dianhong Wang, and Wei Liu, "Compressive Background Modeling for Foreground Extraction," Journal of Electrical and Computer Engineering, vol. 2015. DOI: $10.1155 / 2015 / 295428$.

[28] LIN, Dazhen, CAO, Donglin, et ZENG, Hualin. Improving motion state change object detection by using block background context. In : Computational Intelligence (UKCI), 2014 14th UK Workshop on. IEEE, 2014. p. 1-6. DOI: 10.1109/UKCI.2014.6930187.

[29] ELHARROUSS, Omar, MOUJAHID, Driss, et TAIRI, Hamid. Motion detection based on the combining of the background subtraction and the structure-texture decomposition. Optik-International 
Journal for Light and Electron Optics, 2015, vol. 126, no 24, p. 5992-5997.DOI: 10.1016/j.ijleo.2015.08.084

[30] O. E. Harrouss, D. Moujahid and H. Tairi, "Motion detection based on the combining of the background subtraction and spatial color information," Intelligent Systems and Computer Vision (ISCV), 2015, Fez, 2015, pp. 1-4. DOI: 10.1109/ISACV.2015.7105548.

[31] O. ELHarrouss, D. Moujahid, S. E. Elkaitouni and H. Tairi, "Moving objects detection based on thresholding operations for video surveillance systems," 2015 IEEE/ACS 12th International Conference of Computer Systems and Applications (AICCSA), Marrakech, 2015, pp. 1-5. DOI: 10.1109/AICCSA.2015.7507180. 